\title{
De Novo Transcriptome Analysis of Plant Pathogenic Fungus Myrothecium roridum and Identification of Genes Associated with Trichothecene Mycotoxin Biosynthesis
}

\author{
Wei Ye, Taomei Liu, Muzi Zhu, Weimin Zhang *, Haohua Li, Zilei Huang and Saini Li \\ State Key Laboratory of Applied Microbiology Southern China, Guangdong Provincial Key Laboratory of \\ Microbial Culture Collection and Application, Guangdong Open Laboratory of Applied Microbiology, \\ Guangdong Institute of Microbiology, No. 100 Xianlie Middle Road, Yuexiu District, Guangzhou 510070, China; \\ yewei@gdim.cn (W.Y.); 1tm840801@163.com (T.L.); zhumuzi@foxmail.com (M.Z.); \\ Lihh@gdim.cn (H.L.); yaoyz@gdim.cn (Z.H.); lisn@gdim.cn (S.L.) \\ * Correspondence: wmzhang@gdim.cn; Tel.: +86-20-8768-8309
}

Academic Editor: Dilip Shah

Received: 31 December 2016; Accepted: 17 February 2017; Published: 25 February 2017

\begin{abstract}
Myrothecium roridum is a plant pathogenic fungus that infects different crops and decreases the yield of economical crops, including soybean, cotton, corn, pepper, and tomato. Until now, the pathogenic mechanism of $M$. roridum has remained unclear. Different types of trichothecene mycotoxins were isolated from $M$. roridum, and trichothecene was considered as a plant pathogenic factor of $M$. roridum. In this study, the transcriptome of $M$. roridum in different incubation durations was sequenced using an Illumina Hiseq 2000. A total of 35,485 transcripts and 25,996 unigenes for M. roridum were obtained from $8.0 \mathrm{~Gb}$ clean reads. The protein-protein network of the M. roridum transcriptome indicated that the mitogen-activated protein kinases signal pathway also played an important role in the pathogenicity of $M$. roridum. The genes related to trichothecene biosynthesis were annotated. The expression levels of these genes were also predicted and validated through quantitative real-time polymerase chain reaction. Tri5 gene encoding trichodiene synthase was cloned and expressed, and the purified trichodiene synthase was able to catalyze farnesyl pyrophosphate into different kinds of sesquiterpenoids.Tri4 and Tri11 genes were expressed in Escherichia coli, and their corresponding enzymatic properties were characterized. The phylogenetic tree of trichodiene synthase showed a great discrepancy between the trichodiene synthase from M. roridum and other species. Our study on the genes related to trichothecene biosynthesis establishes a foundation for the $M$. roridum hazard prevention, thus improving the yields of economical crops.
\end{abstract}

Keywords: Myrothecium roridum; plant pathogenic fungus; transcriptome; trichothecene biosynthesis; MAPK signal pathway

\section{Introduction}

Myrothecium roridum is a plant pathogenic fungus widespread in the soils of tropical and subtropical regions. M. roridum infects different economical crops, including soybean, eggplant, pepper, tomato, and cotton, decreasing their yields [1]. This fungus shows strong infectivity, and the spread of M. roridum infection is very fast. Currently, no effective treatment and hazard prevention measure for M. roridum exists. Trichothecenes are sesquiterpene compounds isolated from different kinds of fungi, including Stachybotrys atra [2], Trichoderma brevicompactum [3], and M. roridum [4]. Trichothecenes are the main plant pathogenic factors of Fusarium graminearum; the treatment of trichothecene mycotoxin with wheat could cause gibberellic disease, and a substantial amount of trichothecene mycotoxin was 
found in infected wheat ears [5-8]. However, the main pathogenic mechanism of M. roridum remains unclear. Elucidating the molecular mechanism for the pathogenicity of M. roridum by transcriptome sequencing is necessary to facilitate the prevention of diseases caused by M. roridum.

The genes related to the trichothecene biosynthesis in Fusarium species were identified [9], and the trichodiene synthase encoded by the Tri5 gene was considered a key enzyme in trichothecene biosynthesis. The structural and mechanism analysis of engineered trichodiene synthase from Trichoderma harzianum has been investigated, demonstrating that this enzyme could catalyze the conversion of farnesyl pyrophosphate (FPP) to trichodiene [10]. The Tri5-deleted F. graminearum almost lost its infectivity toward wheat [7]. The Tri6 gene, encoding transcriptional proteins, could regulate trichothecene mycotoxin biosynthesis [6]. The Tri4 gene encoded trichodiene oxygenase, the Tri11 gene encoded isotrichodermin C-15 hydroxylase. The Tri12 gene encoded a kind of membrane transport protein responsible for the exportation of trichothecene mycotoxins [11]. The Tri5 gene from M. roridum was inserted into pMD18T vector and sequenced [12], but this gene has not been heterologously expressed and enzymatic characterized. However, genes related to trichothecene mycotoxin biosynthesis in $M$. roridum have not been fully identified.

Transcriptome sequencing in different kinds of infected plants or pathogenic fungi was performed to investigate the pathogenic mechanisms of fungi [13]. In this study, the transcriptome of M. roridum was sequenced using the Illumina sequencing platform 2000. Genes related to trichothecene mycotoxin biosynthesis in M. roridum including Tri4, Tir5 and Tri11 were identified. This is the first attempt to characterize the complete transcriptome of $M$. roridum and reveal genes related to trichothecene mycotoxin biosynthesis, which would provide a useful theoretical guide for the hazard prevention of $M$. roridum toward economical crops. Moreover, the elucidation of genes related to trichothecene mycotoxin biosynthesis establishes a foundation for the prevention of crop diseases caused by M. roridum.

\section{Results}

\subsection{Sequencing and De Novo Assembly}

In summary, $44,992,952$ sequence reads with Q20 of $92.49 \%$ and GC percent of $57.57 \%$ were generated, and a total of 8,997,597,886 (8.79 GB) nucleotides were obtained (Table 1). Moreover, raw data were deposited in NCBI with accession number SRP080968. The Trinity software (http:/ / trinityrnaseq.sourceforge.net/) was used to assemble short reads through a step-wise strategy, which yielded 5,247,474 contigs with a mean length of $39.83 \mathrm{bp}$ (Figure 1A) and 25,996 unigenes with a mean length of $1094 \mathrm{bp}$ (Figure 1B). A total of 2299 unigenes with lengths greater than $3000 \mathrm{bp}$ were found, indicating the abundance of secondary metabolites in M. roridum.

Table 1. Raw data of $M$. roridum transcriptome.

\begin{tabular}{ccccccc}
\hline Sample ID & Readsum & Basesum & GC (\%) & N (\%) & Q20 (\%) & Q30 (\%) \\
\hline T01 & 44992952 & 8997597886 & 57.57 & 0.01 & 92.49 & 85.07 \\
\hline
\end{tabular}



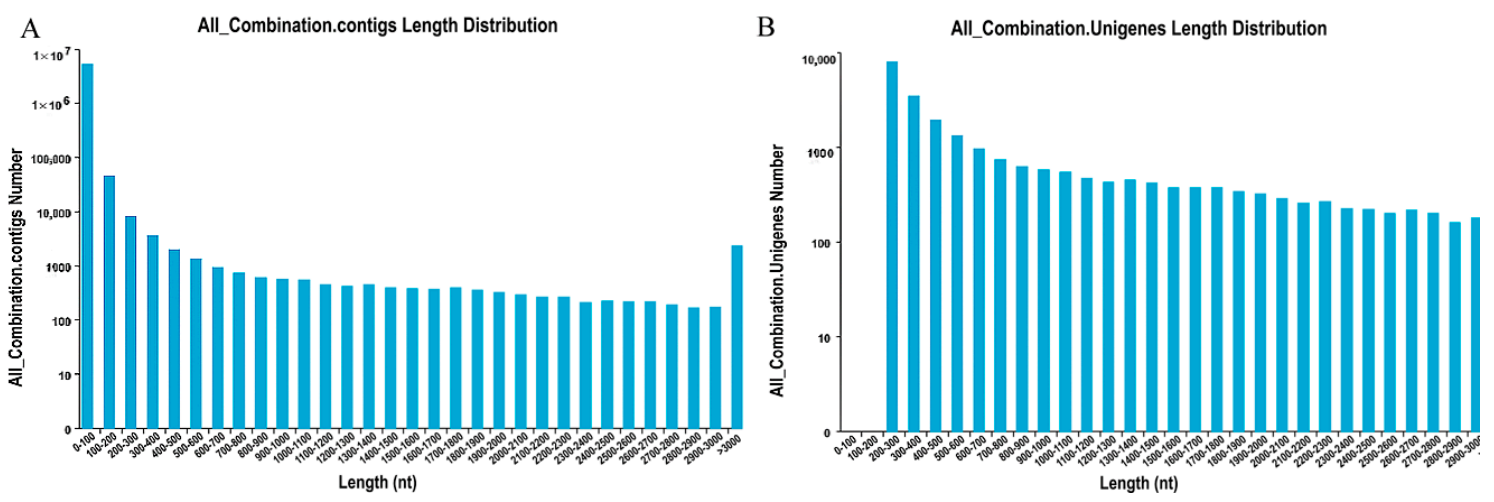

Figure 1. Raw data of M. roridum transcriptome: (A) the distribution of contigs in M. roridum transcriptome; and (B) the distribution of unigenes in M. roridum transcriptome.

\subsection{Functional Annotation and Classification}

All the unigenes were annotated by public databases, including Nr, Nt, Swissprot, KEGG (Kyoto Encyclopedia of Genes and Genomes), COG (Cluster of Orthologous Groups of proteins), GO (Gene Ontology Consortium); 18,199 unigenes were annotated, while 7797 unigenes remained unannotated. A total of 18,160 unigenes matched sequences in the $\mathrm{Nr}$ databases. Furthermore, the unigene number of $M$. roridum annotated in different public databases is shown in Table 2.

Table 2. All unigenes of M. roridum transcriptome annotated in different databases.

\begin{tabular}{cccc}
\hline Anno_Database & Annotated_Number & $\mathbf{3 0 0} \leq$ Length $<\mathbf{1 0 0 0}$ & Length $\geq \mathbf{1 0 0 0}$ \\
\hline COG_Annotation & 7046 & 2026 & 3842 \\
GO_Annotation & 10,775 & 3400 & 4730 \\
KEGG_Annotation & 4569 & 1361 & 2134 \\
Swissprot_Annotation & 11,376 & 3158 & 6458 \\
nr_Annotation & 18,160 & 6034 & 8127 \\
All_Annotated & 18,199 & 6046 & 8128 \\
\hline
\end{tabular}

A total of 10,113 unigenes were annotated and grouped into 25 functional classifications (Figure 2A). The most frequently identified classes were "general function" (2073, 20.5\%), followed by transcription (1024, 10.1\%), carbohydrate transport and metabolism (950, 9.4\%), translation, ribosomal structure and biogenesis (709, 7.0\%), inorganic ion transport and metabolism $(704,6.9 \%)$, secondary metabolites biosynthesis, transport, and catabolism $(696,6.8 \%)$, and post-translational modification, protein turnover, and chaperones (509, 5.0\%). The nuclear structure $(1,0.001 \%)$ and extracellular structure $(0,0 \%)$ categories showed the fewest corresponding genes. Another 10,775 unigenes were annotated by GO database and classified into the biological process, cellular component, and molecular function categories (Figure 2B). The high percentage of unigenes involved in the function of catalytic activity indicated the variety of the secondary metabolites produced by $M$. roridum. To investigate the biological function of unigenes further, a total of 4569 unigenes were assigned to the metabolic pathways described in the KEGG database, including metabolic pathways, biosynthesis of secondary metabolites, ubiquitin-mediated proteolyis, mitogen-activated protein kinase (MAPK) signaling pathway, and regulation of autophagy RNA transport. 


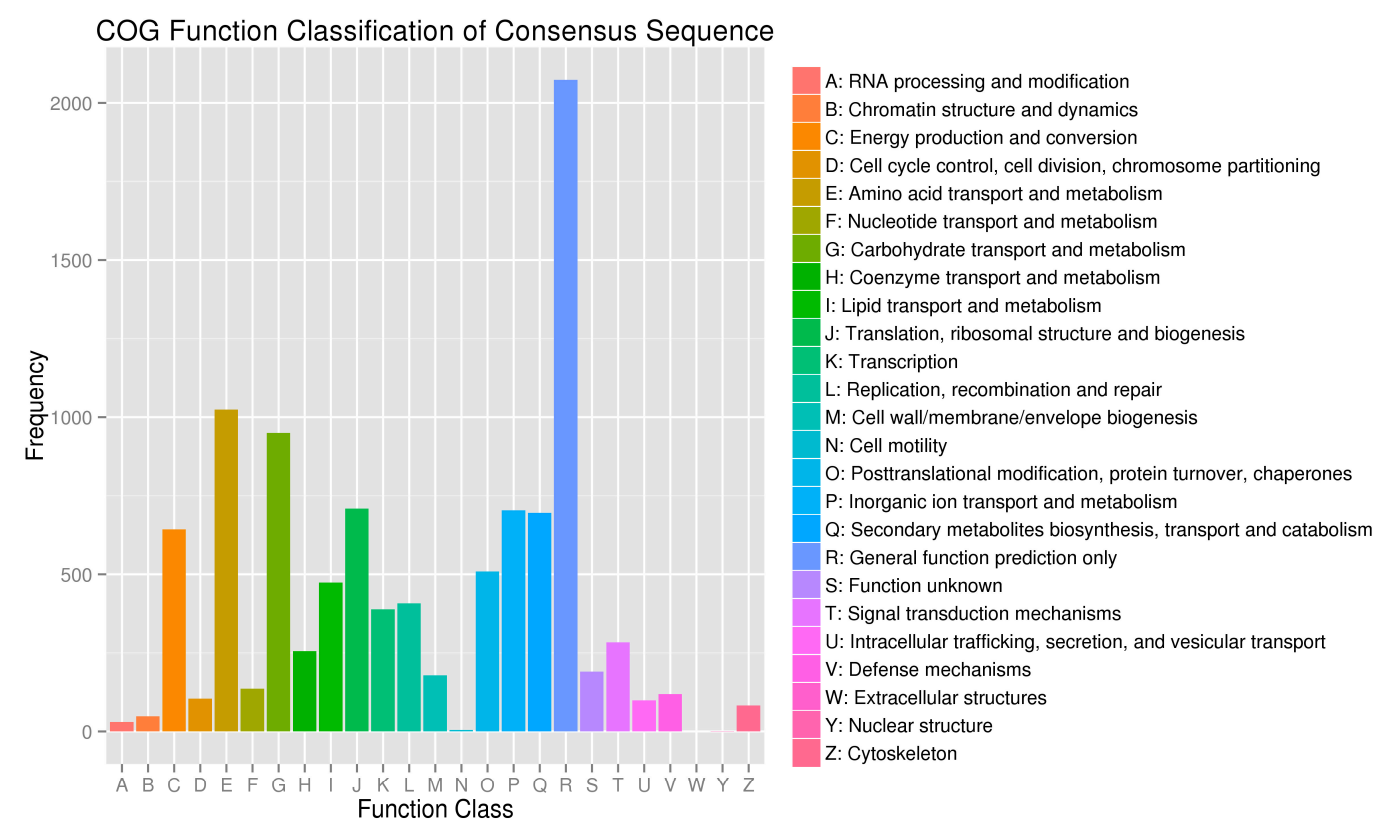

(A)

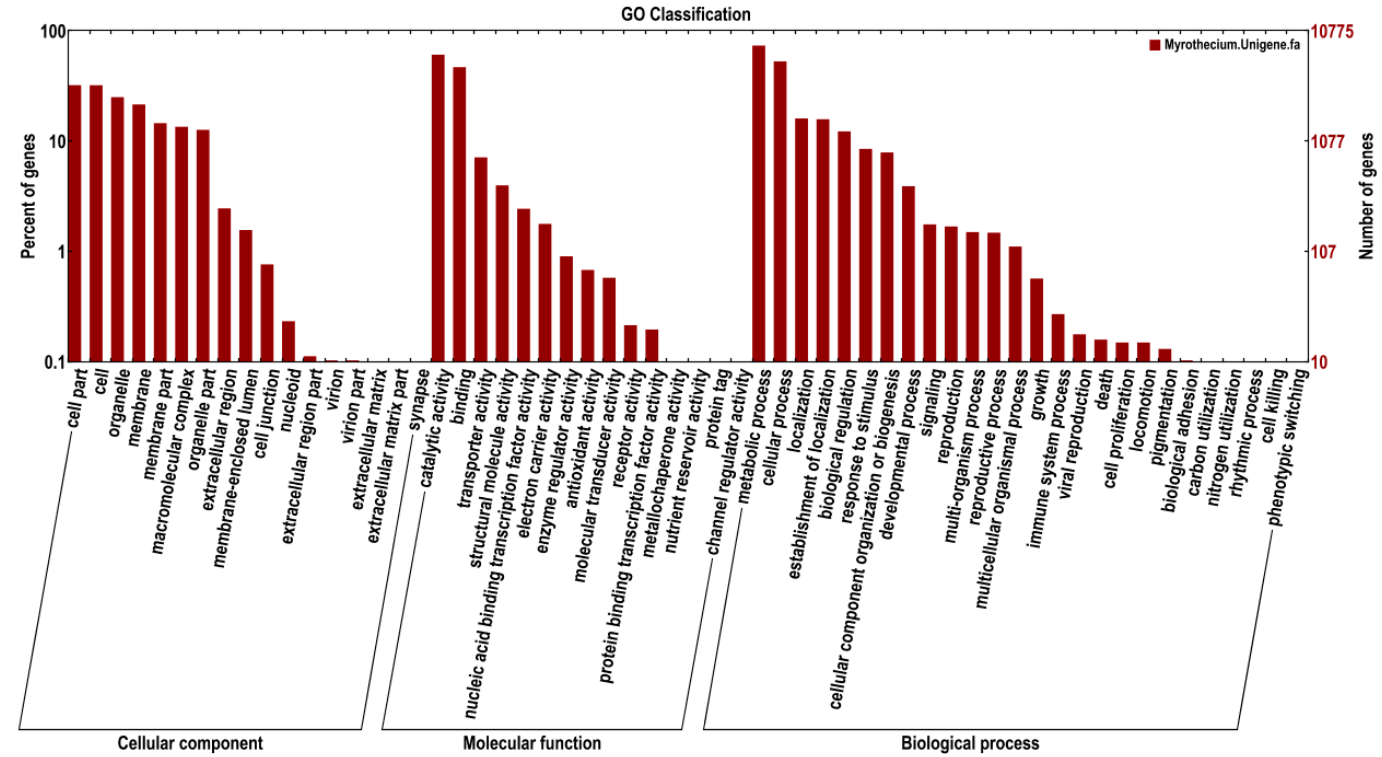

(B)

Figure 2. COG function and GO function classification of the M. roridum transcriptome. (A) COG functional classification of the $M$. roridum transcriptome; (B) GO functional classification of the M. roridum transcriptome.

\subsection{Candidate Genes Involved in Trichothecene Biosynthesis and Expression Analysis of Eight Candidate Genes by $q P C R$}

Trichothecene was postulated to play a very important role in the pathogenicity of M. roridum [4]. Thus the identification of genes involved in trichothecene biosynthesis establishes a foundation for the elucidation of the pathogenic mechanism of $M$. roridum. Trichothecenes are sesquiterpene compounds, and the sesquiterpene and terpenoid backbone biosynthesis pathways in M. roridum are shown in Figure 3. Genes involved in trichothecene mycotoxin biosynthesis are summarized in Table S1. The expression levels of unigenes, including Tir3 (trichothecene 3-O-acetyltransferase), Tri4 (trichodiene oxygenase), Tri5 (trichodiene synthase), Tri6 (trichothecene biosynthesis transcription 
factor), Tri12 (trichothecene efflux pump), and Tri11 (trichothecene 15-O-acetyltransferase) were predicted according to the FPKM (Fragments Per Kilobase of exon model per Million mapped reads) value and validated by qRT-PCR (Quantitative real-time polymerase chain reaction) using primers (Table S2) according to the ORFs of the aforementioned genes (Figure 4A,B). Tri5 gene showed the highest expression level, followed by the genes Tri12, Tri6, and Tri11, suggesting the important roles of these four genes in trichothecene biosynthesis, while GAPDH was used as a reference gene. The expression levels identified by qRT-PCR were in accordance with the prediction. The qPCR products of Tri3, Tri4, Tri5, Tri6, Tri11, and Tri12 genes were detected by agarose gel (Figure $4 \mathrm{C}$ ). The sequencing results indicated that the fragments were desired genes involved in trichothecene biosynthesis.

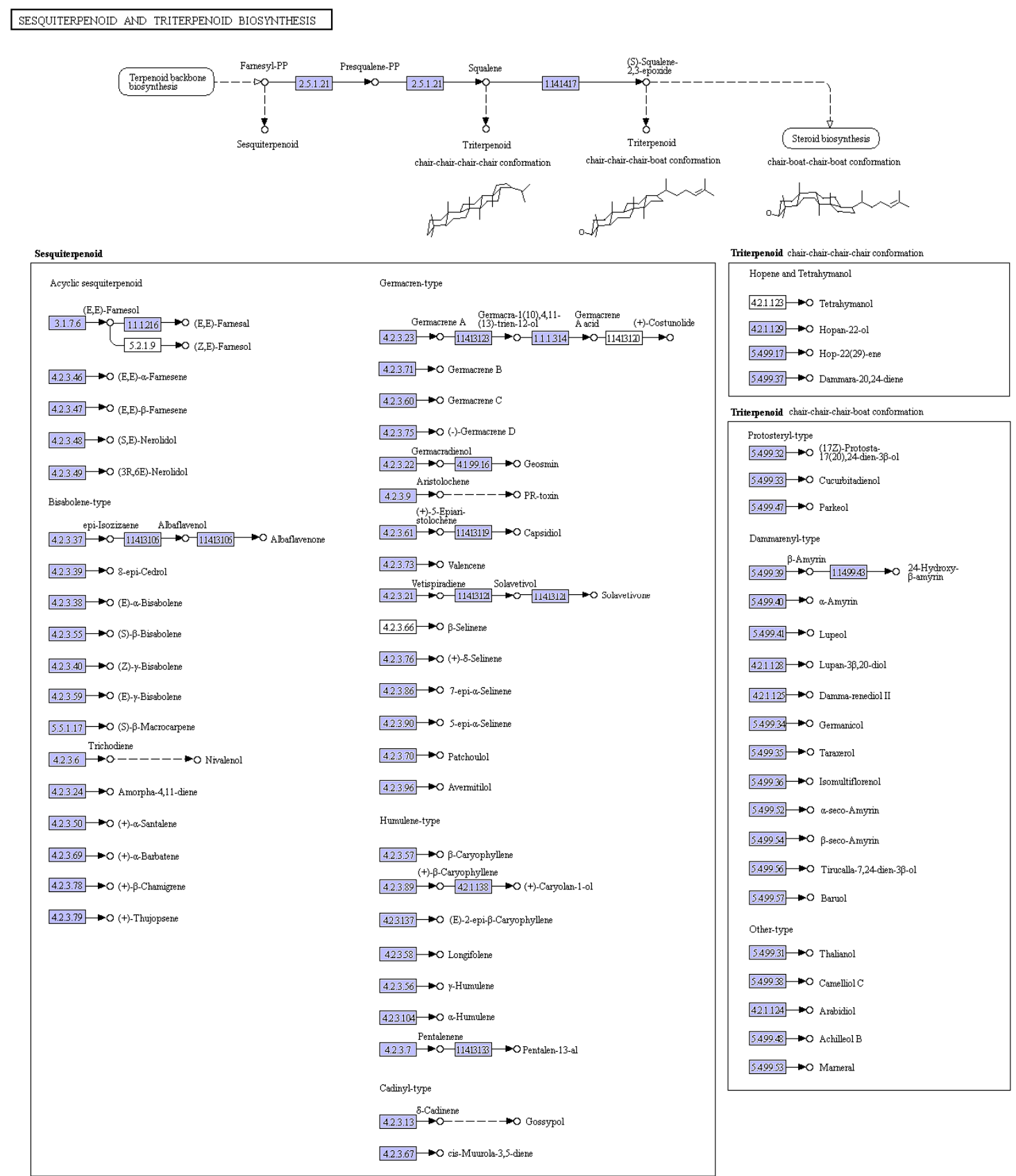

Figure 3. Sesquiterpene and terpenoid backbone biosynthesis pathway in M. roridum. 

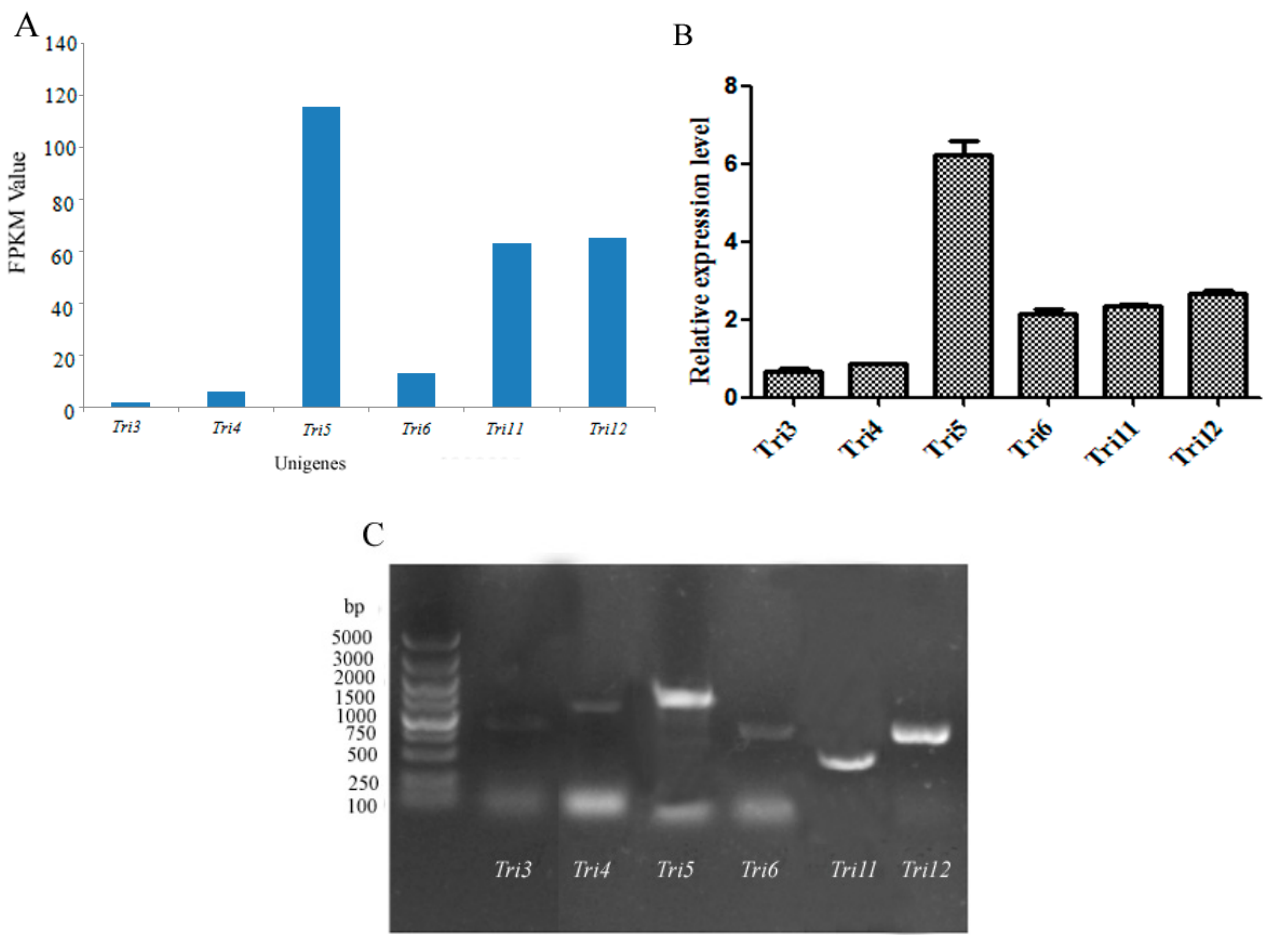

Figure 4. Identification of unigenes involved in trichothecene biosynthesis in M. roridum: (A) prediction of expression levels of unigenes involved in trichothecene biosynthesis according to the FPKM value; (B) validation of expression levels of unigenes involved in trichothecene biosynthesis by qRT-PCR;

(C) agarose electrophoresis of qRT-PCR products of unigenes involved in trichothecene biosynthesis.

\subsection{Identification of Tri4, Tri5, and Tri11 Genes}

Specific primers were designed to amplify the Tri4, Tri5, and Tri11 genes with restriction enzyme sites (NdeI and Xhol), then the amplified fragment was inserted into vector pET28a and expressed in Escherichia coli BL21 (DE3) with a molecular weight of 52.0, 45.0, and $50.0 \mathrm{kDa}$, respectively, which was in accordance with the theoretical value. The trichodiene synthase was purified by Ni affinity chromatography with a purity of $91.9 \%$ (Figure 5A). The Western blot analysis result using anti-His monoclonal antibody confirmed the successful expression and purification of the Tri5 gene (Figure 5B). Trichodiene synthase from $M$. roridum encoded by Tri5 gene were added to the substrate FPP, and the catalyzation products were detected by GC-MS (Gas Chromatograph-Mass Spectrometer), and the sesquiterpenoids including $\beta$-farnesene, nerolidol and farnesol were detected, nerolidol was detected as a main product, which accounted for $68.2 \%$ of all the catalyation products (Figure 6). The result further demonstrated that the trichodiene synthase encoded by Tri5 was a sesquiterpene synthase.

The trichodiene oxygenase encoded by Tri4 from M. roridum (TRO-MR) and isotrichodermin C-15 hydroxylase encoded by Tri11 from M. roridum (ITH-MR) were purified by Ni affinity chromatography with purities of $92.5 \%$ and $95.7 \%$, respectively, which was also demonstrated by Western blot analysis (Figure 7A,B). The substrate thioanisole was used to investigate the enzymatic properties of enzymes encoded by Tri4 and Tri11. Trichodiene oxygenase showed the highest enzymatic activity of $11.26 \pm 1.53 \mathrm{U} / \mathrm{mg}$ towards thioanisole at $30^{\circ} \mathrm{C}$, whereas isotrichodermin $\mathrm{C}-15$ hydroxylase showed the highest enzymatic activity of $31.0 \pm 0.6 \mathrm{U} / \mathrm{mg}$ towards thioanisole at $25^{\circ} \mathrm{C}$ (Figure $7 \mathrm{C}$ ). The optimal reaction $\mathrm{pH}$ values of trichodiene oxygenase and isotrichodermin $\mathrm{C}-15$ hydroxylase from $\mathrm{M}$. roridum with thioanisole were 8.8 (Figure 7D). The enzymatic kinetics of TRO-MR and ITH-MR towards thioanisole was investigated by using different substrates (Figure 7E). ITH-MR and TRO-MR showed $V_{\max }$ and $K_{\mathrm{m}}$ values of $94.34 \mu \mathrm{mol} / \mathrm{mg} \cdot \mathrm{min}, 7.19 \mathrm{mM}$, and $71.43 \mu \mathrm{mol} / \mathrm{mg} \cdot \mathrm{min}, 15.53 \mathrm{mM}$, respectively. The results indicated that ITH-MR showed higher substrate-binding affinity than TRO-MR. 
A $\mathrm{kD}$

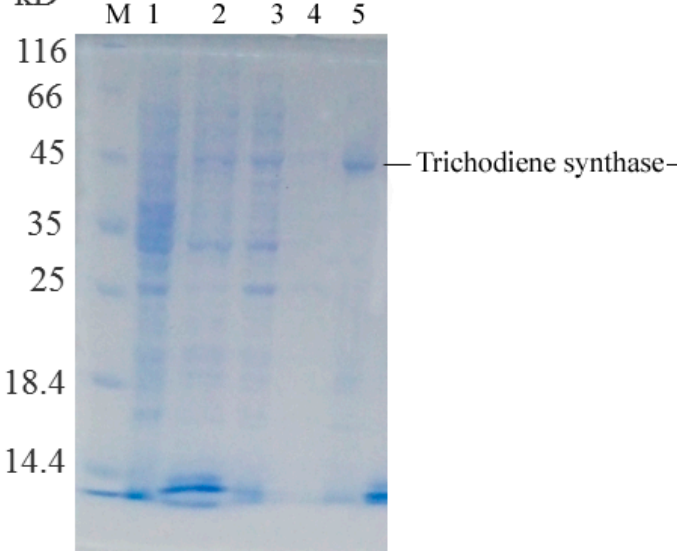

B

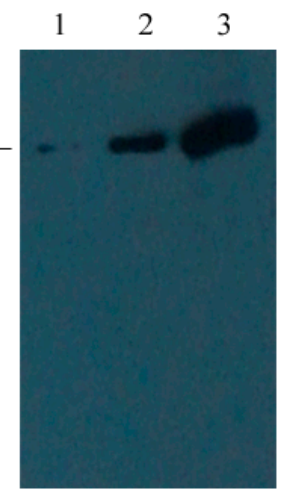

Figure 5. Identification of Tri5 gene: (A) expression and purification of trichodiene synthase encoded by Tri5 gene from M. roridum: M. Protein Marker; 1 . Un-induced sample; 2 . Total proteins of induced samples; 3. Supernatant of induced sample; 4. $55 \mathrm{mM}$ imidazole eluate; and 5. $100 \mathrm{mM}$ imidazole eluate; and (B) Western blot analysis of trichodiene synthase encoded by Tri5 gene from M. roridum: 1 . Total proteins of un-induced sample; 2. Supernatant of induced sample; and 3. $55 \mathrm{mM}$ imidazole eluate.

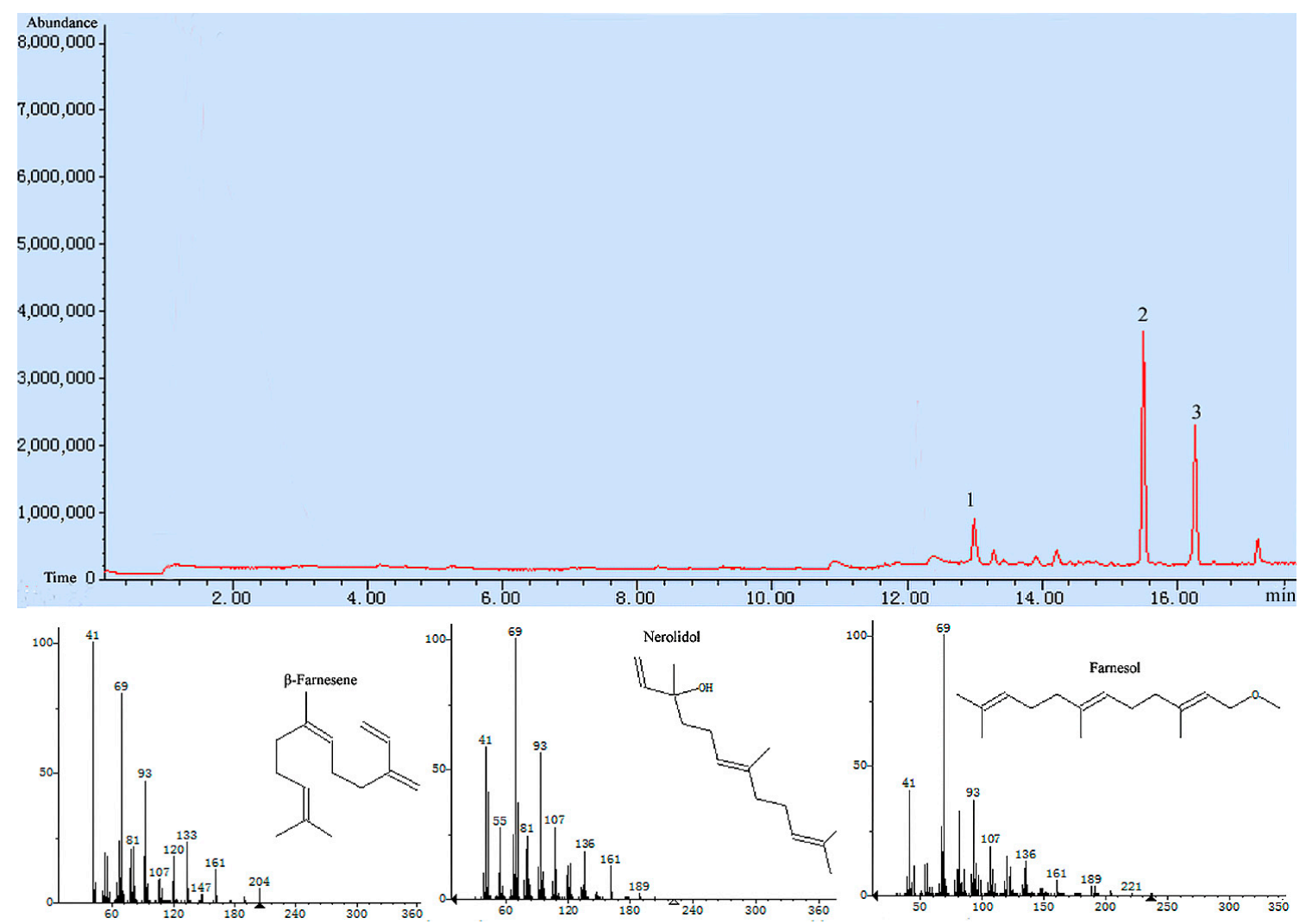

Figure 6. Products catalzyed by trichodiene synthase from M. roridum were detected by GC-MS: peaks 1,2 , and 3 corresponded to $\beta$-farnesene, nerolidol and farnesol, respectively. 
A

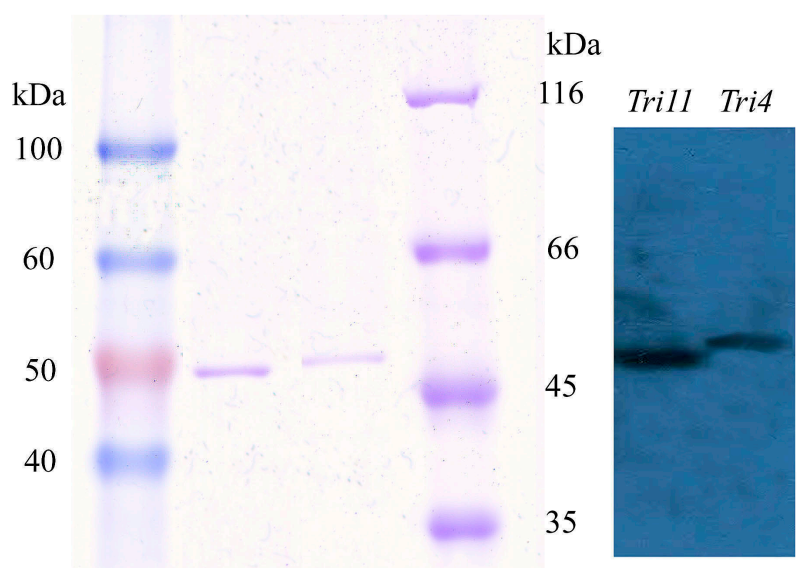

30

20

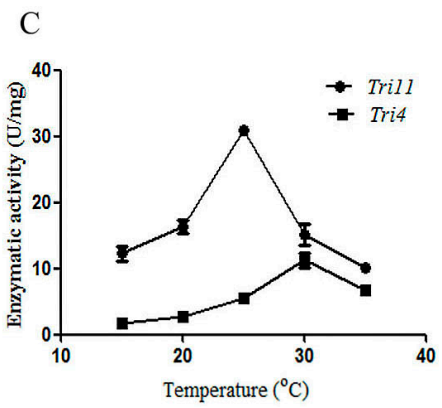

$\mathrm{D}$

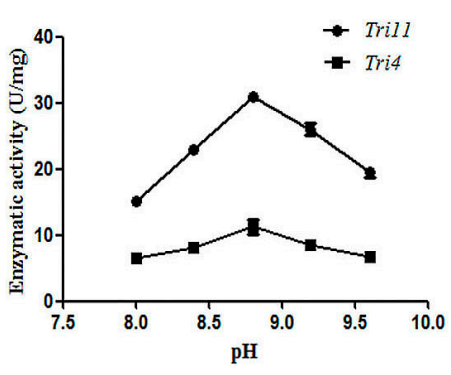

B

$\mathrm{kD}$

Tril1 Tri4

25

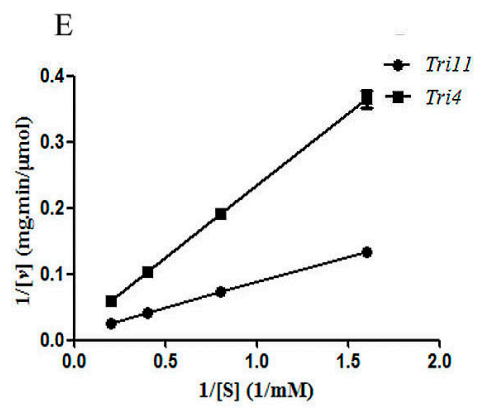

Figure 7. Identification of Tri4 and Tri11 genes: (A) the expression and purification of enzymes encoded by Tri4 (TRO-MR) and Tri11 (ITH-MR), M indicated protein marker; (B) the Western blot analysis of TRO-MR and ITH-MR using anti-His monoclonal antibody; (C) the optimal reaction temperatures of TRO-MR and ITH-MR; (D) the optimal reaction $\mathrm{pH}$ values of TRO-MR and ITH-MR; and (E) the enzymatic kinetics of TRO-MR and ITH-MR, the $K_{\mathrm{m}}$ and $V_{\max }$ values were calculated.

\subsection{Development and Characterization of cDNA-Derived SSR Markers}

Different unigenes in M. roridum were identified by MISA analysis, and 2200 unigenes containing SSR (Simple Sequence Repeat) were identified, among which, 402 sequences contained more than one SSR. Moreover, 202 SSRs were present in the compound formation. The distribution and frequency of the mono-, di-, tri-, tetra-, and hexa-nucleotide repeats were analyzed. The most abundant repeat motifs were mononucleotides $(1115,50.68 \%)$, followed by trinucleotides $(592,26.91 \%)$, dinucleotides (216, 9.82\%), and tetra-nucleotides (48, 2.18\%). The most frequent was A/T $(904,41.09 \%)$, followed by C/G (340, 15.45\%), AGC/CTG (143, 6.5\%), AAAC/GTTT (129, 5.86\%), AG/CT (128, 5.82\%), AAG/CTT (125, 5.68\%), AGG/CTT (91, 4.14\%), and ACG/CGT (72, 3.27\%).

\subsection{Phylogenetic Analysis of Genes Involved in Trichothecene Mycotoxin Biosynthesis}

Tri5 gene, encoding trichodiene synthase in $M$. roridum, played a very important role in trichothecene biosynthesis. Therefore, phylogenetic analysis was conducted using an alignment of all known predicted protein sequences in the NCBI database (Figure S1A), and the result demonstrated that the highest similarity of trichodiene synthase from M. roridum (c10283) with that from Fusarium sporotrichioides was only $26 \%$, indicating significant differences between the trichodiene 
synthase from M. roridum and the trichodiene synthases from other fungal species. Trit was annotated as the trichodiene oxygenase from F. sporotrichioides in the Nr database. It shared a similarity of $32 \%$ with the cytochrome P450 protein from Eutypa lata, and nine kinds of trichodiene oxygenases were found in M. roridum, exhibiting low similarities (Figure S1B). The alignment results demonstrated the diversity and novelty of trichodiene oxygenases from $M$. roridum.

\subsection{Construction of Protein-Protein Interaction Network}

The network of protein-protein interaction involved in the MAPK signaling pathway and ribosome biogenesis in $M$. roridum was constructed (Figure 8). A total of 54 and 17 unigenes were involved in the MAPK signaling pathway and ribosome biogenesis, respectively. The two pathways played very important roles in the growth and virulence of M. roridum. Unigene c10594 encoding U3 small nucleolar RNA-associated protein 7 is the central unigene for the network of ribosome biogenesis in M. roridum. The Unigene 10626 encoding CDC42-GTP-binding protein of the Ras superfamily, unigene c12312 encoding ubiquitin, and unigene c11112 encoding transport protein SEC23 are the central unigenes for the network of MAPK signaling pathway in M. roridum.

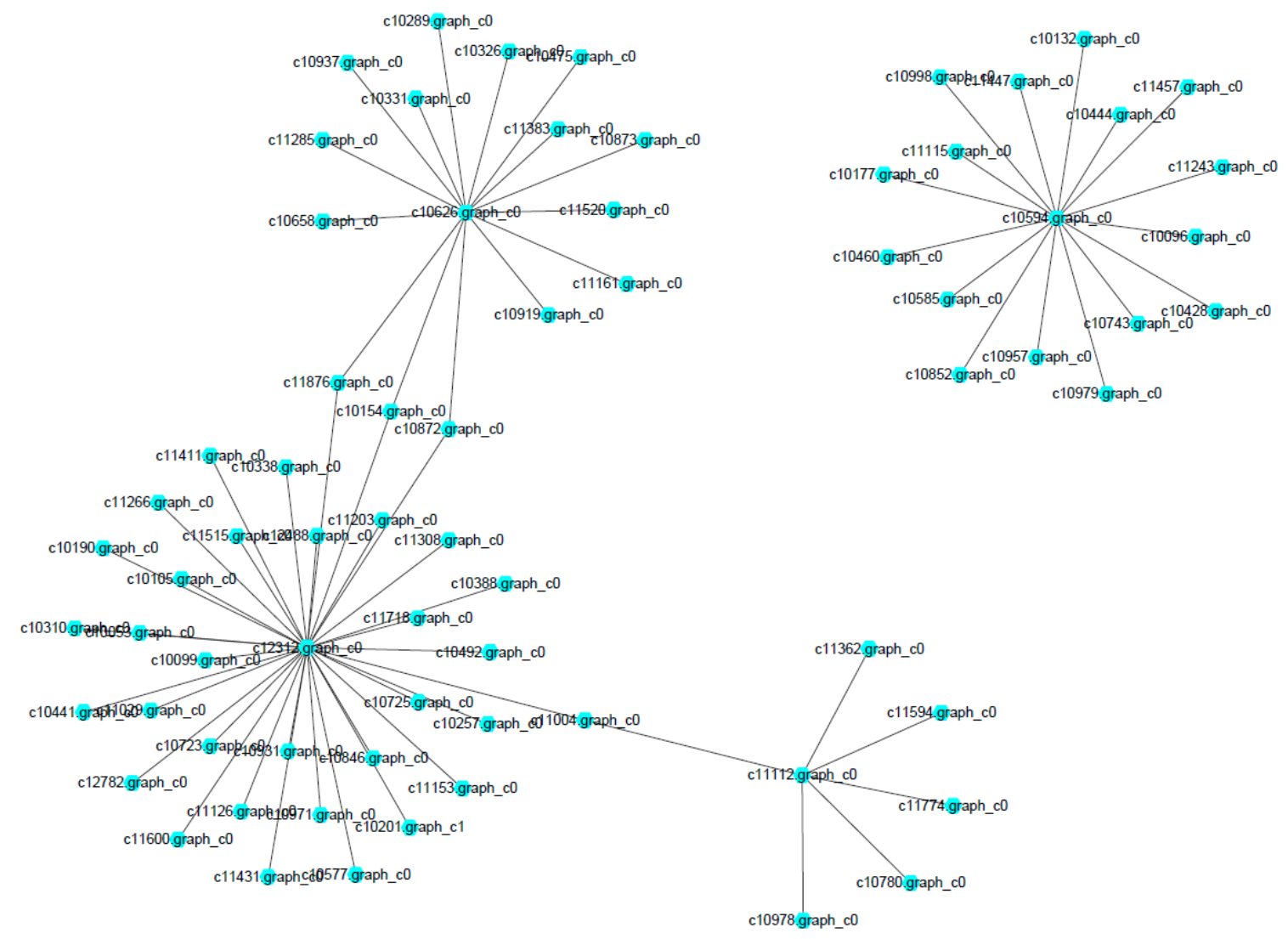

Figure 8. Protein interaction networks in M. roridium transcriptome.

\section{Discussion}

M. roridum is a plant pathogenic fungus that causes great loss to economical crops. The pathogenic mechanism remains unclear. Fungi of Collecotrichum, Nemania, Xylaria, Phomopsis, and Alternaria isolated from leaves of Ageratina adenophora showed strong pathogenicity towards 11 kinds of native plants and four types of economical crops [14]. Phyllosticta capitalensis, a kind of plant endophyte, displayed pathogenicity towards various plant families [15]. The transcriptome analysis is an important approach to reveal the molecular pathology of plant pathogenic fungi. Rhizoctonia solani is an important plant pathogen, which cause disease in many crops as well as ornamental plants and forest trees. 
The transcriptome analysis of R. solani and the identification of pathogenicity-related genes revealed that genes encoding cellulose, pectin and lignin degrading enzymes and genes related to the MAPK pathway played important roles in the pathogenicity of $R$. solani [13].

In our previous work [16], three trichothecene compounds were obtained from the fermentation liquid of M. roridum. In this study, de novo transcriptome analysis of M. roridum isolated from Pogostemon cablin was performed to investigate the pathogenic mechanism and genes associated with trichothecene mycotoxin biosynthesis. A total of 4569 unigenes were assigned to 104 KEGG pathways, and 202 SSRs were identified. Our M. roridum transcriptome results revealed that 47 unigenes, which encoded key enzymes in the trichothecene biosynthesis, including Tri3, Tri4, Tri5, Tri6, Tri11, and Tri12, were postulated according to the biosynthesis pathway of trichothecene mycotoxins in Fusarium spp. qPCR analysis was employed to validate the expression levels of these unigenes. PCR and sequencing were performed to confirm the results of the transcriptome analysis. qRT-PCR analysis results revealed that Tri5 gene showed the highest expressed level, Tri12, Tri6 genes took the second and the third positions, respectively. These results demonstrated the important role of Tri5 gene in the biosynthesis of trichothecene mycotoxins. The phylogenetic analysis of genes Tri4 and Tri5 revealed that significant differences between the trichothecene-biosynthesis-related unigenes from $M$. roridum and those from other fungal species existed. The identification of Tri5 gene from $M$. roridum confirmed that the function of Tri5-encoding protein as a sesquiterpene synthase, meanwhile, trichothecene mytoxins are known as a kind of sesquiterpenoids, thus demonstrating the important role of Tri5 gene in the biosynthesis of trichothecene mytoxins. The identification of Tri4 and Tri11 genes from M. roridum demonstrated the function of Tri4 and Tri11 as monooxygenase, which played an important role in the biosynthesis of trichothecene mytoxins. The transcriptome analysis of $M$. roridum and identification of the genes related to trichothecene biosynthesis establishes a foundation for the elucidation of molecular plant pathology of the M. roridum.

Eleven unigenes, which were found in the transcriptome of $M$. roridum, were annotated as Tri4 gene encoding trichodiene oxygenase. Different Trit unigenes showed significant differences between each other, indicating that different kinds of trichodiene oxygenases in M. roridum contributed to the biosynthesis of different secondary metabolites. Epiroridin acid, epiroridin E, and mytoxin B were isolated from the fermentation liquid of $M$. roridum [16] (Figure S2), and trichodiene oxygenase was assumed to play an important role in the transformation of epiroridin E into mytoxin B. Only one unigene encoding trichodiene synthase was annotated in the M. roridum transcriptome, and this trichodiene synthase showed the highest similarity of $26 \%$ with that from the F. sporotrichioides, suggesting that unigene $\mathrm{c} 3810$ encodes a novel trichodiene synthase. The three trichothecene compounds, epiroridin E, epiroridin acid, and mytoxin B, isolated from M. roridum showed different structures with known trichothecene mycotoxins, such as T-2 and DON toxin, indicating the possible special biosynthesis mechanism of trichothecens in M. roridum. Moreover, the amino acid sequence of regulatory protein encoded by the Tri6 gene from M. roridum showed the highest similarity of $65 \%$ with that from F. sporotrichioides, implying the possibly different regulatory mechanism of trichothecene biosynthesis in M. roridum.

The stel2 gene involved in the MAPK signal pathway in opportunistic pathogenic fungus Cryptococcus neoformans was reportedly capable of regulating the expression of virulence-related genes, including capsule and melanin-related genes $[17,18]$, regulating the virulence of $C$. neoformans. MAPK signal pathway was also reported to be involved in the process of phenotype switching and mycelium formation, which played an important role in the invasive infection of Candida albicans [19]. Thus, the MAPK signal pathway, especially the transport protein SEC23, is assumed to have played an important role in the pathogenicity of $M$. roridum. 


\section{Materials and Methods}

\section{1. cDNA Library Construction and RNA Sequencing}

The strain M. roridum A553 (Accession No. KJ813720) was isolated from the medicinal plant Pogostemon cablin, a kind of Guangdong medicinal plant collected from Xinyi City, Guangdong province. M. roridum was inoculated on a PDA medium and incubated at $30^{\circ} \mathrm{C}$ for $3,5,7$, and 9 days. The total RNAs of $M$. roridum at different growth stages were determined using the RNA extracting kit (Umagen, Guangzhou, China). The quantity, purity, and integrity of RNA were checked on a $1.5 \%(w / v)$ agarose gel and the Nanodrop-2000 spectrophotometer (GE, Fairfield, CT, USA). HPLC (High Performance Liquid Chromatography) Agilent 2100 (Agilent, Santa Clara, CA, USA) was used to detect the RIN value of the total RNA. High-quality samples (RNA $\geq 6.0$ ) were selected for high-throughput sequencing. Then, the different RNAs were mixed with the same amount. Total RNAs in an amount of $5.0 \mu \mathrm{g}$ were resuspended in RNase free-water and stored at $-80{ }^{\circ} \mathrm{C}$ until use. The extracted RNA samples were used for the complementary DNA (cDNA) synthesis. Poly(A) mRNA was isolated using oligo-dT beads (Qiagen, Germantown, MD, USA). All mRNAs were broken into short fragments (200 nt) by adding a fragmentation buffer. First-strand cDNA was generated using random hexamer-primed reverse transcription, followed by the synthesis of the second-strand cDNA using RNase $\mathrm{H}$ and DNA polymerase I. The cDNA fragments were purified using a QIAquick PCR extraction kit (Qiagen, Germantown, MD, USA). These purified fragments were then washed with Elution buffer for end reparation poly $(\mathrm{A})$ addition and ligated to sequencing adapters. Following the agarose gel electrophoresis and extraction of cDNA from gels, the cDNA fragments (200 $\pm 25 \mathrm{bp})$ were purified and enriched by PCR to construct the final cDNA library. The cDNA library was sequenced on the Illumina sequencing platform (Illumina HiSeq ${ }^{\mathrm{TM}}$ 2000, Illumina, San Diego, CA, USA) using the single-end paired-end technology in a single run. The original image processes of sequencing, base-calling, and quality value calculation were performed by the Illumina GA Pipeline (version 1.6, Illumina, San Diego, CA, USA), in which 90-bp paired-end reads were obtained [20,21].

\subsection{Data Processing, Assembly, and Annotation}

Transcriptome sequencing was conducted using the Illumina $\mathrm{HiSeq}^{\mathrm{TM}} 2000$ sequencing platform. To assemble the entire transcriptomes of the different samples better, a paired-end (PE)100 sequencing strategy was used. All sequences were examined to ensure their accuracy. A Perl program was written to select clean reads by removing low-quality sequences (more than $50 \%$ bases with quality lower than 20 in one sequence and Q30 less than 80\% were identified), reads with more than 5\% N bases (bases unknown), and reads containing adaptor sequences. Subsequently, the clean reads were assembled using the Trinity software (version 1.4, Campton, NH, USA) to construct unique consensus sequences. Adaptor and low-quality sequences were trimmed. Short sequences $(<50 \mathrm{bp})$ were removed using a custom Perl program (version 5.14, USA). The resulting high-quality sequences were deposited in the National Center for Biotechnology Information (NCBI) database and de novo assembled into contigs and transcripts. To reduce data redundancy, transcripts with a minimum length of $200 \mathrm{bp}$ were assembled and clustered using the CLC NGS Cell software (version 1.3, Illumina, San Diego, CA, USA) under default parameters. The longest sequences in each cluster were reserved and designated as unigenes. Searches were performed using local BLASTX programs (NCBI, NIH, Bethesda, MD, USA) against sequences in the NCBI non-redundant (nr) protein database and the SWISS-PROT database (the $e$ value cut-off was $1 \times 10^{-5}$ ) [22]. Unigenes were tentatively identified according to top hits against known sequences. The resulting unigenes were used as references for the determination of GO and COG terms and were analyzed further using the KEGG database.

\subsection{KEGG Pathway Analysis and Predicted CDS}

Pathway assignments were made according to KEGG mapping. Enzyme commission numbers were assigned to unique sequences that had the best BLASTX scores with cutoff $e$ values of $1.00 \times 10^{-5}$, 
as determined from our KEGG database search. The KEGG database is capable of analyzing gene products during the metabolism process and related gene function in the cellular processes. The sequences were mapped to the KEGG biochemical pathways according to the Enzyme Commission (EC) distribution within the pathway database.

Then, we used blast results information to extract CDS (Coding Domain Sequences) from Unigene sequences and translated them into peptide sequences. Moreover, blast results information was also used to train ESTScan [23]. The CDS of unigenes that had no hit in BLAST were predicted by ESTScan and then translated into peptide sequences.

\subsection{Quantitative Real-Time Polymerase Chain Reaction Analysis}

To verify the quality of the sequences assembled in this study, unigenes related to the biosynthesis of trichothecene were validated by quantitative real-time polymerase chain reaction (qRT-PCR). qRT-PCR was performed using the Mastercycler ep realplex System (Eppendorf, Westbury, NY, USA) with 2SYBR Green mix (Fermentas, Harrington, DE, USA) according to the manufacturer's instructions. First-strand cDNA was synthesized from $1 \mu \mathrm{g}$ of total RNA with reverse transcriptase (Takara, Tokyo, Japan) and oligo (dT) 15 primer, and the resulting products were used as templates for qRT-PCR. The specific primers used for qRT-PCR are listed in Table S2. The qRT-PCR thermal cycling condition for all reactions was $95^{\circ} \mathrm{C}$ for $1 \mathrm{~min} 50 \mathrm{~s}$, followed by 40 cycles at $95{ }^{\circ} \mathrm{C}$ for $10 \mathrm{~s}$ and $55^{\circ} \mathrm{C}$ for $33 \mathrm{~s}$. All reactions were conducted in triplicates, and the results were expressed as relative expression levels to the GAPDH gene. The $C_{t}$ values obtained were used as the original data to calculate the relative expression level of different genes to histone gene by the $2^{-\Delta \Delta C t}$ method $[24,25]$. Each sample was analyzed in triplicate.

\subsection{Identification of Tri4, Tri5, and Tri11 Genes}

The Tri4, Tri5, and Tri11 genes were amplified with restriction enzymes NdeI and XhoI using primers listed in Table S3, then inserted into the expression vector pET28a and transformed with E. coli, and the recombinant vector pET28a-Tri4, pET28a-Tri5, and pET28a-Tri11 were expressed in E. coli BL21 (DE3) after being induced using $1 \mathrm{mM}$ of IPTG for $3.0 \mathrm{~h}$. A supernatant of $200 \mathrm{~mL}$ fermentation liquid containing pET28a-Tri5 after sonication was loaded onto the $\mathrm{Ni}$ affinity chromatography. The target protein was eluted with $20 \mathrm{mM}$ Tris- $\mathrm{HCl}(\mathrm{pH}$ 8.0) containing different concentrations of imidazole. Different protein samples were identified by sodium dodecyl sulfate-polyacrylamide gel electrophoresis and transferred to the NC membrane. After being blocked by $5 \%$ non-fat milk, the membrane was incubated with mouse anti-His monoclonal antibody (Earthox, Millbrae, CA, USA) and goat anti mouse IgG antibody (Promega, Madison, WI, USA), and target bands were finally visualized using an ECL kit (Fermentas, Harrington, DE, USA) following the manufacturer's instructions.

\subsection{Enzymatic Activity Assay of Enzymes Encoded by Tri4, Tri5, and Tri11}

Briefly, farnesyl pyrophosphate (FPP) substrate with a concentration of $46 \mu \mathrm{M}$ and $50 \mu \mathrm{L}$ Tri5 encoding-protein with a concentration of $0.6 \mathrm{mg} / \mathrm{mL}$ were added to a buffer containing $25 \mathrm{mM}$ Tris-HCl (pH 7.0), $100 \mathrm{mM} \mathrm{MgSO} 4,10 \%$ glycerol, and 5 mM DTT. A $200 \mu \mathrm{L}$ aliquot of the mixture was immediately sealed and incubated at $37^{\circ} \mathrm{C}$ for $1 \mathrm{~h}$. A solid phase extraction column (Anpel, Shanghai, China) was used to absorb sesquiterpenes produced by catalysis of Tri5 encoding-protein at $65^{\circ} \mathrm{C}$ for $0.5 \mathrm{~h}$. The absorbed products were loaded onto a HP 6890/5975C GC-MS apparatus (Agilent, Santa Clara, CA, USA). The GC-MS running procedure is as following: the injection port temperature is $250{ }^{\circ} \mathrm{C}$, the initial temperature is $80^{\circ} \mathrm{C}$, increased to $180^{\circ} \mathrm{C}$ with a gradient of $5^{\circ} \mathrm{C} / \mathrm{min}$, then increased to $250{ }^{\circ} \mathrm{C}$ with a gradient of $20^{\circ} \mathrm{C} / \mathrm{min}$. The flow rate of helium is $1.0 \mathrm{~mL} / \mathrm{min}$. The structure and abundance of the produced sesquiterpenes were analyzed.

NADPH with content of $0.40 \mu \mathrm{mol}$ and $0.04 \mathrm{mg}$ enzymes were added into substrate thioanisole with a concentration of $1.0 \mu \mathrm{mol}, 50 \mathrm{mM}$ Tris- $\mathrm{HCl}(\mathrm{pH} 8.8)$ was added to total volume of $200 \mu \mathrm{L}$. 
The mixture was incubated at $25^{\circ} \mathrm{C}$, the increase or the decrease in absorbance at $340 \mathrm{~nm}$ were monitored. $1 \mathrm{U}$ was defined as $1 \mu \mathrm{mol} \mathrm{NADPH}$ was consumed in one minute. The optimal reaction temperature and reaction $\mathrm{pH}$ for trichodiene oxygenase encoded by Tri4 and isotrichodermin $\mathrm{C}-15$ hydroxylase encoded by Tri11 were investigated under the reaction temperatures of $15^{\circ} \mathrm{C}, 20^{\circ} \mathrm{C}, 25^{\circ} \mathrm{C}$, $30^{\circ} \mathrm{C}, 35^{\circ} \mathrm{C}$ and reaction $\mathrm{pH}$ values of 8.0, 8.4, 8.8, 9.2, and 9.6. Substrate thioanisole with different concentrations of $5.0 \mathrm{mM}, 2.5 \mathrm{mM}, 1.25 \mathrm{mM}$, and $0.625 \mathrm{mM}$ were used to investigate the enzymatic kinetics of enzymes encoded by Tri4 and Tri11 genes.

\subsection{Phylogenetic Analysis of Genes Related to Trichothecene Mycotoxin Biosynthesis}

A phylogenetic tree was constructed with MEGA version 5.0 (Arizona State University, Tempe, AZ, USA) using the neighbor-joining method [26]. NCBI and the transcriptome of $M$. roridum were searched for different trichodiene synthases and trichodiene oxygenases. The functional interaction networks of proteins were integrated using the STRING (Search Tool for the Retrieval of Interacting Genes/Proteins) database with the confidence parameter set at 0.15 threshold. The expression clusters of the CsWRKY genes from each cultivar were analyzed using Cluster (http://bonsai.hgc.jp/ mdehoon/software/ cluster/software.htm). A diagram was drawn using Tree View (http://jtreeview.sourceforge.net/).

\subsection{Identification of SSRS}

The MIcroSAtellite identification tool (MISA, http://pgrc.inpk-gatersleben.de/misa/) was used to identify SSRs [27]. The parameters were adjusted to identify perfect dinucleotide, trinucleotide, tetranucleotide, pentanucleotide, and hexanucleotide motifs with a minimum of 6 , 5, 5, 4, and 4 repetitions, respectively. Primer pairs were designed using the Primer 3 software and selected according to the following criteria: (1) primers with SSRs were eliminated; (2) primers aligned with unigene sequences were allowed three mismatches at the $5^{\prime}$ site and one mismatch at the $3^{\prime}$ site; (3) primers that aligned to more than one unigene were eliminated; and (4) SSRs were identified using the ssr_finder (http:/ / www.fresnostate.edu/ssrfinder/). We kept the products whose results from ssr_finder and MISA were the same.

\section{Conclusions}

In conclusion, the transcriptome of $M$. roridum was first sequenced by an Illumina Hiseq 2000 system. The expression levels of genes related to the trichothecene biosynthesis were analyzed, and several genes related to the biosynthesis of trichothecene were cloned and expressed. The results demonstrated that Tri5 gene played an important role in the biosynthesis of trichothecene mycotoxins in M. roridum. The enzymatic properties investigation results demonstrated that Tri4 and Tri11 genes' function as monooxygenase in the biosynthesis of trichothecene mycotoxins. The protein-protein network of the M. roridum transcriptome indicated that the MAPK signal pathway also played a role in the pathogenicity of $M$. roridum. The above results establish a foundation for the elucidation of the trichothecene biosynthesis and molecular mechanism of $M$. roridum pathogenicity, thus providing a molecular basis for controlling $M$. roridum infection through genetic engineering approaches.

Supplementary Materials: Supplementary materials can be found at www.mdpi.com/1422-0067/18/3/497/s1.

Acknowledgments: Financial support for this research was provided by the Basic Research Program of China (973 Program) (No. 2014CB460613), National Natural Science Foundation of China (31500037, 31600271), Natural Science Foundation of Guangdong Province (2015A030313710, 2015A030310103), Science and Technology Planning Project of Guangdong Province (2015A030302060, 2016A020222022, 2015A030302061), and the Talent Foundation of Guangdong Academy of Sciences (No. 2017GDASCX-0823).

Author Contributions: Wei Ye and Weimin Zhang conceived and designed the experiments; Wei Ye, Taomei Liu, Muzi Zhu, Saini Li and Zilei Huang performed the experiments; Wei Ye and Weimin Zhang analyzed the data; Haohua Li and Saini Li contributed materials; and Wei Ye and Weimin Zhang wrote the paper.

Conflicts of Interest: The authors declare no conflict of interest. 


\section{Abbreviations}

$\begin{array}{ll}\text { CDS } & \text { Coding Domain Sequences } \\ \text { COG } & \text { Cluster of Orthologous Groups of proteins } \\ \text { FPKM } & \text { Fragments Per Kilobase of exon model per Million mapped reads } \\ \text { FPP } & \text { Farnesyl pyrophosphate } \\ \text { GC-MS } & \text { Gas Chromatograph-Mass Spectrometer } \\ \text { GO } & \text { Gene Ontology Consortium } \\ \text { HPLC } & \text { High Performance Liquid Chromatography } \\ \text { ITH-MR } & \text { Isotrichodermin C-15 hydroxylase from } M \text {. roridum } \\ \text { KEGG } & \text { Kyoto Encyclopedia of Genes and Genomes } \\ \text { MAPK } & \text { Mitogen-activated protein kinase } \\ \text { qRT-PCR } & \text { Quantitative real-time polymerase chain reaction } \\ \text { SSR } & \text { Simple Sequence Repeat } \\ \text { STRING } & \text { Search Tool for the Retrieval of Interacting Genes/Proteins } \\ \text { TRO-MR } & \text { Trichodiene oxygenase from } M \text {. roridum }\end{array}$

\section{References}

1. Hong, C.F.; Tsai, S.F.; Yeh, H.C.; Fan, M.C. First report of Myrothecium roridum causing Myrothecium leaf spot on Dieffenbachia picta "Camilla" in Taiwan. Plant Dis. 2013, 97, 1253. [CrossRef]

2. Jarvis, B.B.; Lee, Y.W.; Comezoglu, S.N.; Yatawara, C.S. Trichothecenes produced by Stachybotrys atra from Eastern Europe. Appl. Environ. Microbiol. 1986, 51, 915-918. [PubMed]

3. Nielsen, K.F.; Grafenhan, T.; Zafari, D.; Thrane, U. Trichothecene production by Trichoderma brevicompactum. J. Agric. Food Chem. 2005, 53, 8190-8196. [CrossRef] [PubMed]

4. Bean, G.A.; Fernando, T.; Jarvis, B.B.; Bruton, B. The isolation and identification of trichothecene metabolites from a plant pathogenic strain of Myrothecium roridum. J. Nat. Prod. 1984, 47, 727-729. [CrossRef] [PubMed]

5. Wang, Y.Z.; Miller, J.D. Effects of Fusarium graminearum metabolites on wheat tissue in relation to Fusarium head blight resistance. J. Phytopathol. 1988, 122, 118-125. [CrossRef]

6. Proctor, R.H.; Hohn, T.M.; McCormick, S.P. Reduced virulence of Gibberella zeae caused by disruption of a trichothecene toxin biosynthetic gene. Mol. Plant Microbe Interact. 1995, 8, 591-601. [CrossRef]

7. Proctor, R.H.; Hohn, T.M.; McCormick, S.P.; Desjardins, A.E. Tri6 encodes an unusual zinc finger protein involved in regulation of trichothecene biosynthesis in Fusarium sporotrichioides. Appl. Environ. Microbiol. 1995, 61, 1923-1930. [PubMed]

8. Proctor, R.H.; Hohn, T.M.; McCormick, S.P. Restoration of wild-type virulence to Tri5 disruption mutants of Gibberella zeaevia gene reversion and mutant complementation. Microbiology 1997, 143, 2583-2591. [CrossRef] [PubMed]

9. Desjardins, A.E.; Hohn, T.M.; McCormick, S.P. Trichothecene biosynthesis in Fusarium species: Chemistry, genetics, and significance. Micobiol. Mol. Biol. Rev. 1993, 57, 595-604.

10. Kumari, I.; Chaudhary, N.; Sandhu, P.; Akhter, Y. Structural and mechanistic analysis of engineered trichodiene synthase enzymes from Trichoderma harzianum: Towards higher catalytic activities empowering sustainable agriculture. J. Biomol. Struct. Dyn. 2016, 34, 1176-1189. [CrossRef] [PubMed]

11. Alexander, N.J.; McCormick, S.P.; Hohn, T.M. TRI12, a trichothecene efflux pump from Fusarium sporotrichioides: Gene isolation and expression in yeast. Mol. Genet. Genom. 1999, 261, 977-984. [CrossRef]

12. Trapp, S.C.; Hohn, T.M.; Mccormick, S.; Jarvis, B.B. Characterization of the gene cluster for biosynthesis of macrocyclic trichothecenes in Myrothecium roridum. Mol. Genet. Genom. 1998, 257, 421-432. [CrossRef]

13. Wibberg, D.; Jelonek, L.; Rupp, O.; Krober, M.; Goesmann, A.; Grosch, R.; Puhler, A.; Schluter, A. Transcriptome analysis of the phytopathogenic fungus Rhizoctonia solani AG1-IB 7/3/14 applying high-throughput sequencing of expressed sequence tags (ESTs). Fungal Biol. 2014, 118, 800-813. [CrossRef] [PubMed]

14. Mei, L.; Zhu, M.; Zhang, D.Z.; Wang, Y.Z.; Guo, J.; Zhang, H.B. Geographical and temporal changes of foliar fungal endophytes associated with the invasive plant Ageratina adenophora. Microb. Ecol. 2014, 67, 402-409. [CrossRef] [PubMed] 
15. Wikee, S.; Lombard, L.; Crous, P.W.; Nakashima, C.; Motohashi, K.; Chukeatirote, E.; Alias, S.A.; Mckenzie, E.H.; Hyde, K.D. Phyllosticta capitalensis, a widespread endophyte of plants. Fungal Divers. 2013, 60, 91-105. [CrossRef]

16. Liu, H.X.; Liu, W.Z.; Chen, Y.C.; Sun, Z.H.; Tan, Y.Z.; Li, H.H.; Zhang, W.M. Cytotoxic trichothecene macrolides from the endophyte fungus Myrothecium roridum. J. Asian Nat. Prod. Res. 2016. [CrossRef]

17. Kojima, K.; Bahn, Y.S.; Heitman, J. Calcineurin, Mpk1 and Hog1 MAPK pathways independently control fludioxonil antifungal sensitivity in Cryptococcus neoformans. Microbiology 2006, 152, 591-604. [CrossRef] [PubMed]

18. Huang, Y.M.; Zhu, H.M.; Wen, H. A role of MAPK pathway in the pathogenic mechanism of Cryptcococcus neoformans. Chin. J. Mycol. 2012, 5, 304-308.

19. Wang, D.; Huang, X.; Zhao, L.L.; Zhao, J.J. Advances in the MAPK signaling pathway of Candida albicans. Chin. J. Mycol. 2013, 8, 252-256.

20. Li, P.; Deng, W.Q.; Li, T.H.; Song, B.; Shen, Y.H. Illumina-based de novo transcriptome sequencing and analysis of Amanita exitialis basidiocarps. Gene 2013, 532, 63-71. [CrossRef] [PubMed]

21. Gao, J.; Yu, X.; Ma, F.; Li, J. RNA-Seq analysis of transcriptome and glucosinolate metabolism in seeds and sprouts of broccoli (Brassica oleracea var. italica). PLoS ONE 2014, 9, e88804. [CrossRef] [PubMed]

22. Wu, J.K.; Zhang, W.Q.; Huang, S.B.; He, Z.Q.; Cheng, Y.B.; Wang, J. SOAP fusion: A robust and effective computational fusion discovery tool for RNA-seq reads. Bioinformatics 2013, 29, 2971-2978. [CrossRef] [PubMed]

23. Chen, X.R.; Brurberg, M.B.; Elameen, A.; Klemsdal, S.S.; Martinussen, I. Expression of resistance gene analogs in woodland strawberry (Fragaria vesca) during infection with Phytophthora cactorum. Mol. Genet. Genom. 2016, 291, 1967-1978. [CrossRef] [PubMed]

24. Huang, L.; Li, G.Y.; Mo, Z.L.; Xiao, P.; Li, J.; Huang, J. De novo assembly of the Japanese flounder (Paralichthys olivaceus) spleen transcriptome to identify putative genes involved in immunity. PLoS ONE 2015, 10, e0117642.

25. Ye, W.; Wu, H.Q.; He, X.; Wang, L.; Zhang, W.M.; Li, H.H. Transcriptome sequencing of chemically induced Aquilaria sinensis to identify genes related to agarwood formation. PLoS ONE 2016, 11, e0155505. [CrossRef] [PubMed]

26. Tamura, K.; Stecher, G.; Peterson, D.; Filipski, A.; Kumar, S. MEGA6: Molecular evolutionary genetics analysis version 6.0. Mol. Biol. Evol. 2011, 30, 2725-2729. [CrossRef] [PubMed]

27. Zhang, M.F.; Jiang, L.M.; Zhang, D.M.; Jia, G.X. De novo transcriptome characterization of Lilium 'Sorbonne'and key enzymes related to the flavonoid biosynthesis. Mol. Genet. Genom. 2015, 290, $399-412$. [CrossRef] [PubMed]

(C) 2017 by the authors. Licensee MDPI, Basel, Switzerland. This article is an open access article distributed under the terms and conditions of the Creative Commons Attribution (CC BY) license (http://creativecommons.org/licenses/by/4.0/). 